\title{
The Research Jigsaw: How To Get Started
}

\author{
Christine M Bond
}

$\mathrm{T}_{\mathrm{n}}^{\mathrm{h}, \mathrm{n}}$ he purpose of this article, the first in our "Research

Primer" series, is to create awareness of the whole research process, including what research is, how to do it, the value of funding, and the need for dissemination. This first article can be regarded as the picture on the top of a jigsaw box, which allows you to see how all the individual jigsaw pieces, each on its own of little value, come together. In a research study, the whole truly is greater than the sum of the parts.

A good starting point is to understand exactly what "research" is. Two important points about research are that it is objective and involves study of a very specific issue. The word "research" has French origins and first appeared in the 16th century. ${ }^{1}$ It means the act of searching closely and carefully; both this and all subsequent definitions incorporate the main principles of systematicity and the generation of new knowledge. As long as these ideas are applied, the exact nature of research can be highly variable, depending on context, purposes, and methods, all of which affect the work that is ultimately undertaken. Research can be purely theoretical, as in astrophysics and the initial theoretical proposal of the Higgs boson particle ${ }^{2}$; it can be laboratory based, such as early biochemical studies; it can involve testing in animals or humans (i.e., applied or clinical research); or it can be purely based on observation, counting, and enquiry (i.e., health services research). Because of the systematic, documented approach, the work can be repeated by others, the extent of generalizability beyond the population under study is understood, and-most importantly - the conclusions are not based on anecdote.

Pharmacy-related research, especially research conducted alongside daily practice, is likely to be on the continuum from clinical to health services research. Thus, the research in which pharmacists engage will probably involve assessing the clinical effectiveness and cost-effectiveness of new treatments (Do they work? Are they safe? Do they represent value for money?), defining current practice and potentially identifying areas of suboptimal care, describing the patient's perspectives and needs, and assessing new methods of service delivery. For example, a study might compare the outcomes of pharmacist prescribing with those of physician prescribing.

In recent years, clinical audit has become a core activity in health care, allowing robust assessment of current practice against predetermined standards. If the clinical audit cycle is completed, an intervention to improve practice is introduced, with subsequent re-auditing to see if the desired improvement has been effected. Audit should use the same rigorous approach as all other research, but its purpose is different, in that it is generally focused on local service improvement and implementation.

Research is important and enjoyable. Here in the United Kingdom, pharmacists are often referred to as "scientists on the high street" (or, as North Americans might say, "scientists on main street"), and as scientists we are all likely to have a naturally analytical and enquiring approach to practice. However, research can also be challenging and frustrating, and it takes time. It is therefore key to undertake research, where possible, on a topic of interest and relevance to you-it should be something you really want to find out about, such that your enthusiasm for the topic will take you through the hard times, should they arise. So think first about your daily practice. Identify the aspects of your practice where you have often asked, "Supposing I did that differently, what would happen? or "Does that drug really work?" Get a few ideas and talk them over with colleagues, both peers and more senior colleagues, to get a sense of the wider importance of your topic and whether it can be turned into a project. It is likely that if you do research in the workplace, you will be involving these colleagues, so it would be helpful if they also believed the topic were relevant and worth exploring. Assuming there is support for your ideas at this stage, you should then do a scoping literature search to see what is already known about the topic, and perhaps collect some data by doing a small, local pilot study. If things still look promising, get ready to start your research in earnest.

One of the key aspects of doing research is articulating an appropriate and specific study question, which can be surprisingly time-consuming. This step is important because the research question and its underpinning aims and objectives will govern the study design. Another reason for having a clear research question is that it focuses activities and, in theory, should prevent enthusiasm from getting the better of logical enquiry, whereby, for example, additional questions are included in a survey "because they seem interesting" rather than because they contribute to answering the main study question. An example of a poorly worded research question would be "What happens when pharmacists prescribe instead of 
physicians?" As stated, this represents an idea to explore, not a research question. An example of a good research question might be "What is the difference in pain outcomes for patients with chronic pain when pharmacists prescribe compared with physicians?" The second version makes it clear that the patient group consists of people with chronic pain, which helps to define the specific clinical outcomes to be studied, and the implication of a comparison with physicians indicates a comparative (ideally randomized), controlled, and thereby prospective study. The topic of articulating the research question is covered in the second article in this series ${ }^{3}$ (see page 31 of this issue).

Specifying the research question appropriately leads on nicely to the next steps in designing the study. First, you must identify the participants, where they are located, and how they can be identified. Continuing with the example of pharmacist prescribing in the setting of chronic pain, one group of participants will be patients with chronic pain who are attending a pain clinic, identified from patient lists. The other group of participants will be the pharmacists and physicians involved in treating these patients. Next, you need to decide what data to collect; in the hypothetical study, these data are likely to include basic demographic information about the patients and their medical history (to aid comparisons across groups receiving either pharmacist or physician prescribing, and to allow subgroup analyses by patient types), as well as pain-related outcomes. These outcomes should ideally be validated measures that have proven to be reliably associated with different levels of pain, such as the Chronic Pain Grade ${ }^{4}$ or the McGill Pain Questionnaire. 5

It is also important at this stage to decide for how long data should be collected, in what format (e.g., questionnaire or interview or observation), and how often. For example, you might use postal questionnaires to collect baseline data, and 3- and 12-month follow-up to assess immediate effects and sustainability of any changes. Given that pharmacist prescribing for patients with chronic pain is a new service that might be implemented, qualitative approaches such as focus groups or interviews would also be good to explore the experiences of patients, pharmacists, and physicians and to collect suggestions for change, if any, to the new service. If you anticipate that this study will inform service change, it might also be important to collect information on the cost-effectiveness of the service. All of these approaches-questionnaire surveys, interviews, focus groups, and health economics analysis-plus other topics will be covered in detail in subsequent articles in the Research Primer series.

The foregoing is a quick overview of the design of a small study. Individual components, such as a questionnaire survey, might be used for a much simpler study, for example, a survey to explore pharmacists' beliefs about their capability to prescribe for patients with chronic pain, or an interview or focus group study involving patients with chronic pain to identify patients' goals in pain management.

Once you and your colleagues on the research team have made all the required decisions about how the research will be conducted, you should be able to write the study protocol. This detailed document is in effect a road map for your project. It will include the background to the project, the research question, the aims and objectives you intend to address, and the details of how you will do it. If you are applying for funding, the study protocol will form the basis of the funding application; it is also an essential component of the application for ethics approval to conduct your work. Applying for ethics approval will also be covered in detail in a later article in this series.

Whatever study design you undertake, the outcome will be data of some sort. It might take the form of narrative transcripts from interviews or focus groups or lots of numbers from surveys. Whichever sort of data you have, they must be stored securely. Increasingly, this means using a software package such as NVivo (www.qsrinternational.com/ products_nvivo.aspx) for qualitative data or SPSS (www01.ibm.com/software/analytics/spss/) for statistical analysis of quantitative data. Subsequent articles in this series will take you through the various types of analyses in more detail. However, one important point to mention at this stage is the necessity of reporting results as informatively as possible. You must turn your data into information that will be accessible to the reader. As an example, Table 1 and Figure 1 show mock data for hospital clinic appointments for chronic pain by age. It should be clear which is the easier to understand!

The final step in conducting research is to report your findings. Without telling people what you have done, you might as well not have done it! Dissemination can be accomplished through formal academic papers submitted to journals, conference presentations in oral or poster format, internal reports, and briefing papers. Each has its strengths and weaknesses, and often all are used to ensure that research results are available to all who might be interested, in a form that is relevant for them. Again, subsequent articles in this series will describe these options in more detail. However, what all approaches to dissemination need is interpretation from you, the researcher, of what you have found. What is your main result, the overarching finding? What are the strengths and limitations of what you have done? How do your findings compare with previous work, and are there implications for practice or policy?

So that's it! Your research—finished and published! A few words of caution are needed at this point. If this is your first project, keep it small and simple. Don't expect your first piece of research to be a randomized trial published in The Lancet. Remember also that small building blocks of data from small, 
Table 1. Number of People Attending Pain Clinic by Age*

\begin{tabular}{|c|c|c|c|c|c|}
\hline Age, yr & $\begin{array}{c}\text { No. of } \\
\text { Admissions }\end{array}$ & Age, yr & $\begin{array}{c}\text { No. of } \\
\text { Admissions }\end{array}$ & Age, yr & $\begin{array}{c}\text { No. of } \\
\text { Admissions }\end{array}$ \\
\hline$\leq 16$ & 0 & 28 & 0 & 40 & 0 \\
\hline 17 & 0 & 29 & 0 & 41 & 0 \\
\hline 18 & 1 & 30 & 0 & 42 & 2 \\
\hline 19 & 0 & 31 & 0 & 43 & 0 \\
\hline 20 & 0 & 32 & 2 & 44 & 3 \\
\hline 21 & 0 & 33 & 0 & 45 & 0 \\
\hline 22 & 1 & 34 & 1 & 46 & 0 \\
\hline 23 & 0 & 35 & 0 & 47 & 1 \\
\hline 24 & 0 & 36 & 1 & 48 & 0 \\
\hline 25 & 0 & 37 & 0 & 49 & 0 \\
\hline 26 & 0 & 38 & 0 & 50 & 0 \\
\hline 27 & 1 & 39 & 1 & Etc. & \\
\hline
\end{tabular}

*Mock data. See Figure 1 for graphic presentation of the same data.

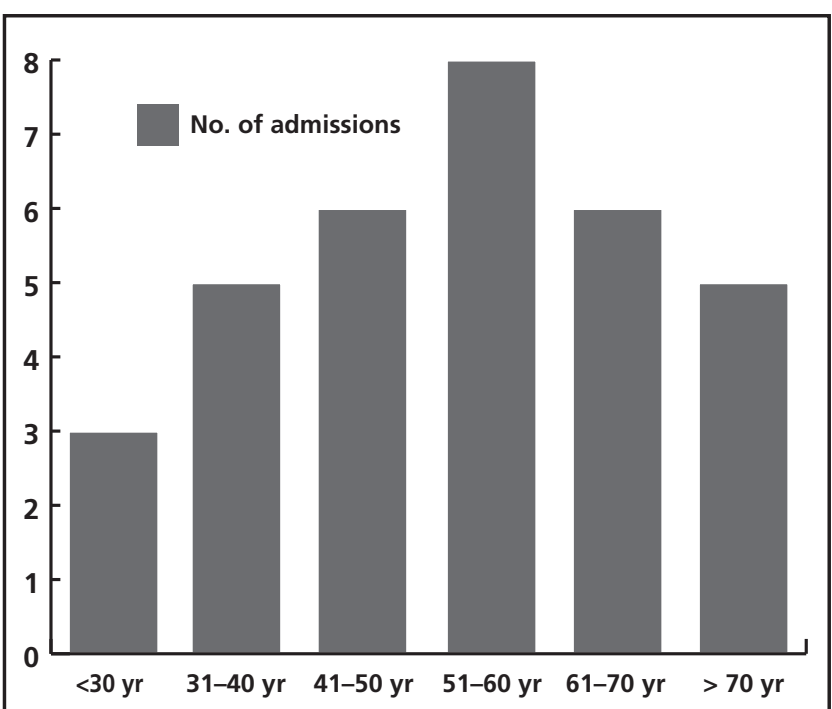

Figure 1. Number of people attending pain clinic by age group. (Mock data. See Table 1 for tabular presentation of part of this dataset.)

well-conducted studies can lead to a bigger, definitive study. Consider getting help with laborious, administrative tasks such as formatting and distributing questionnaires, and transcribing or entering data. This might mean applying for funding but could simply involve asking a student or colleague for a few hours of help. If you don't understand some aspect of the project, be sure to get the expert help you need, by attending training sessions, reading books, searching the web, asking peers, or contacting academic colleagues.

To summarize: find an exciting and relevant topic, define an objective research question, design the study to systematically answer your question, and tell people what you have found - even if it is not what you anticipated!

\section{References}

1. Onions CT. Oxford dictionary of English etymology. Oxford (UK): Clarendon Press; 1966.

2. Cho A. Higgs boson makes its debut after decades-long search. Science. 2012;337(6091):141-3.

3. Tully M. Research: articulating questions, generating hypotheses, and choosing study designs. Can J Hosp Pharm. 2014;67(1):31-4.

4. Smith BH, Penny KI, Purves AM, Munro C, Wilson B, Grimshaw J, et al. The Chronic Pain Grade questionnaire: validation and reliability in postal research. Pain. 1997;71(2):141-7.

5. Melzack R. The McGill Pain Questionnaire: major properties and scoring methods. Pain. 1975;1(3):277-99.

Christine M Bond, BPharm, PhD, MEd, is with the Pharmacy, Centre of Academic Primary Care, University of Aberdeen, Foresterhill, Aberdeen, Scotland. She is also an Associate Editor with the CJHP.

Competing interests: Christine Bond has received reimbursement from Wiley for travel expenses to attend professional meetings and conferences in her role as Editor in Chief of the International Journal of Pharmacy Practice, as well as travel reimbursement from the Canadian Institutes of Health Research to contribute to "Best Brains Exchanges 2012". For research activities outside the scope of this article, she has received multiple research grants (paid to her institution) from European, UK, and Scottish research councils and charities, as well as a grant from Novartis.

\section{Address correspondence to:}

Professor Christine M Bond

Pharmacy

Centre of Academic Primary Care

Room 1.123

Polwarth Building West Block

Foresterhill, Aberdeen AB25 2ZD

Scotland

e-mail: c.m.bond@abdn.ac.uk

This article is the first in the CJHP Research Primer Series, an initiative of the CJHP Editorial Board and the CSHP Research Committee. The planned 2-year series is intended to appeal to relatively inexperienced researchers, with the goal of building research capacity among practising pharmacists. The articles, presenting simple but rigorous guidance to encourage and support novice researchers, are being solicited from authors with appropriate expertise. 